\title{
Is computer aided learning as effective as traditional tutorials?
}

\author{
Teaching periodontal pocket charting to dental students: a comparison of computer assisted learning and \\ traditional tutorials V. Bissell, R. A. McKerlie, D. F. Kinane and S. McHugh Br Dent J 2003; 195: 333-336
}

\section{Aim}

The aim of this study was to compare the effectiveness of a computer assisted learning (CAL) programme with that of traditional small group tutorials in teaching theoretical and practical aspects of periodontal pocket charting.

\section{Method}

Sixty-one third year undergraduate dental students were randomized to either receive a tutorial or to work through the CAL programme. Students using the CAL programme completed questionnaires relating to previous computer experience and the ease of use of the programme. All students were assessed immediately after the intervention by means of a confidence log, a practical exercise and a further confidence log. They were assessed again three weeks later by means of a confidence log and a multiple-choice written test.

\section{Results}

There were very few significant differences between groups for any of the assessments used. However, subjective comments indicated that students occasionally felt disadvantaged if they had not received a tutorial.

\section{Conclusion}

CAL and traditional teaching methods are equally effective in teaching periodontal pocket charting to undergraduate dental students.

\section{IN BRIEF}

- A CAL programme may be as effective as traditional tutorials in some areas of dental teaching.

- Students may value personal interaction with a teacher.

- Formal evaluation of CAL programs helps in their modification and informs decisions regarding their subsequent use.

\section{COMMENT}

In this paper, the authors compared the effectiveness of two different methods of teaching the theoretical and practical aspects of periodontal pocket charting to undergraduate dental students. One method was traditional small group teaching in tutorial format, the other used a CAL programme delivered over the internet.

This was an objective study, with one year of 61 students randomly assigned to one of the two teaching methods. Immediately after the delivery by either format, each student was asked to fill in a confidence log, then undertake a practical test on a custom made model simulator, followed by the confidence log again. Three weeks later, the confidence log was repeated, and immediately followed by a written multiple choice test.

Fifty nine students completed all evaluations, 28 using the CAL programme, 31 taking part in a tutorial. Statistical analysis revealed no significant differences between groups in the practical test, although students from the tutorial group tended to be more confident. There were very few significant differences between groups in the confidence log responses, although again the tutorial group tended to be more confident in understanding how to grade furcation involvement (this result alerted the authors to the need to improve the illustrations in this part of the programme). Performance in the written test revealed no difference between the groups.

At a time when a number of dental schools are looking to review the undergraduate curriculum, and a number of different educational philosophies are being advocated, it is timely to have such a paper comparing two teaching methods objectively. It is perhaps fortunate that there were no significant differences between the groups since half of the year may have felt they had not been taught this particular subject in the best way! When students were asked for their subjective comments, a number expressed concern that they might have been disadvantaged by not receiving a tutorial.

The authors point out that a CAL programme is standardized and consistent, whereas in the study the small group tutorials were given by seven different tutors. Therefore the former would be expected to give better results, and yet the findings do not support this. They suggest caution in interpretation of the results but feel that CAL programmes are ideal for the purposes of revision of concepts.

Mr R. B. Winstanley, Reader in Restorative Dentistry Department of Adult Dental Care, School of Clinical Dentistry, University of Sheffield doi:10.1038/sj.bdj.4810533 